\title{
Karriereentwicklungen von Frauen und Männern in der Medizin
}

\section{B. Buddeberg-Fischer}

\begin{abstract}
Einleitung
Die Medizin durchläuft gegenwärtig tiefgreifende Veränderungen. In der Forschung liefert die sogenannte molekulare Medizin zahlreiche neue und faszinierende Erkenntnisse. In der Krankenversorgung bemühen sich neben Ärztinnen und Ärzten immer mehr Gesundheitsökonomen und -manager, die Kostenentwicklung zu steuern und den Gesundheitsmarkt mit attraktiven Angeboten und neuen Konzepten zu beliefern. Parallel zu diesen Entwicklungen findet ein Wandel statt, den man als «Feminisierung der Medizin" charakterisieren könnte. Immer mehr Männer wenden sich von dem bis anhin prestigeträchtigen Arztberuf ab, während eine zunehmende Zahl von Frauen die Medizin als ein für sie attraktives Berufsfeld entdecken.

Ich möchte im folgenden einigen Aspekten dieses Wandels mit einem Blick in die Vergangenheit, aktuellen Ergebnissen und Analysen aus jüngster Zeit und einigen Gedanken zur Karriereentwicklung junger Ärztinnen und Ärzte in der Zukunft nachgehen.
\end{abstract}

\section{Stellung von Männern und Frauen in der Medizin im historischen Zeitspiegel*}

Obwohl sich Frauen seit jeher in den Gebieten der Frauenheilkunde, der Kinderpflege, der gesunden Lebensführung und der Ernährung aktiv betätigt haben, belegen historische Quellen, dass die Medizin im engeren Sinn bis weit in die Mitte des 20. Jahrhunderts vorwiegend von Männern ausgeübt wurde. Die ärztliche Kunst wurde lange Zeit von sogenannten «Meistern" ihren Lehrlingen weitergegeben und später in Medizinschulen gelehrt. Frauen wurden weder als

\footnotetext{
* Für die Unterstützung bei der Beschaffung des medizin-historischen Quellenmaterials sowie für wichtige Hinweise bei der Bearbeitung des Themas bin ich Herrn Prof. Beat Rüttimann zu grossem Dank verpflichtet.
}

Korrespondenz:

PD Dr. med. Barbara Buddeberg-Fischer

UniversitätsSpital Zürich

Culmannstrasse 8

CH-8091 Zürich

E-mail: barbara.buddeberg@psy.usz.ch
Lehrlinge aufgenommen noch wurde ihnen der $\mathrm{Zu}$ gang zu den Medizinschulen gewährt. Andererseits gab es in früheren Jahrhunderten nicht die strenge Abgrenzung zwischen medizinischen Berufen und medizinischen Hilfsberufen. Diese Unterscheidung setzte erst mit der Akademisierung und Professionalisierung der Medizin im 19. und 20. Jahrhundert ein.

Wie verlief nun die Entwicklung der Medizin in ihren frühen Anfängen?

Hippokrates gilt als Begründer einer wissenschaftlichen Heilkunde. Noch heute sind wir in unserer ärztlichen Tätigkeit dem hippokratischen Eid verpflichtet. Vor allem im Zusammenhang mit der zunehmenden Bedeutung ethischer Fragestellungen in der modernen Medizin finden die bereits im hippokratischen Eid erwähnten ethischen Grundprinzipien (voluntas, nil nocere und iustitia) wieder vermehrte Beachtung.

Im frühen Mittelalter wurde die Heilkunde vor allem in den Klöstern gelehrt und weiterentwickelt. Später kam es mit der Gründung der Universitäten zu einer Aufgliederung der Ärzteschaft: Auf der einen Seite solche mit einer akademischen Ausbildung, die sogenannten "physici» oder Stadtärzte, die man auch als Schriftgelehrte bezeichnen kann, und auf der anderen Seite die Wundärzte, Bader, Scherer und Schröpfer, welche die chirurgischen Eingriffe durchführten. Letztere organisierten sich in den Zünften der Handwerker.

In Basel, wo 1460 die erste Universität der Schweiz gegründet wurde, konnte man schon früh eine akademische Ausbildung zum Arzt erwerben. In Zürich, das erst 1833 eine Universität erhielt, erwarben sich die Söhne einflussreicher Familien zunächst eine chirurgische Ausbildung. Danach gingen sie entweder nach Basel oder an eine ausländische Universität, um die akademische Ausbildung nachzuholen, zu promovieren und dann wieder nach Zürich zurückzukehren. Erst im 19. Jahrhundert verschwand allmählich die Kluft zwischen den Zünften und der Universität. So wurde an der neu gegründeten Zürcher Universität von Anfang an ein chirurgischer Lehrstuhl eingerichtet.

\section{Experiment Frauenstudium an der Universität Zürich}

Die erste Hälfte des 19. Jahrhunderts war in Zürich geprägt von einer liberalen Aufbruchstimmung. An der 1833 neu gegründeten Universität besuchten neben führenden männlichen Vertretern der Zürcher Gesellschaft auch Frauen Abendvorlesungen. 1865 wurde die Russin Nadezhda Suslowa als Hörerin medizinischer Vorlesungen zugelassen und 1867 auf Antrag der Medizinischen Fakultät rückwirkend immatrikuliert, so dass sie ihre Promotion ablegen konnte. Dieser Entscheid kam nicht ganz freiwillig. Bei der Gründung der Universität hatte man im Universitätsreglement nicht ausdrücklich festgehalten, dass das Studium und ein offizieller Abschluss nur dem männlichen Geschlecht vorbehalten sein soll. Das Jahr 1867 bedeutete deshalb einen Meilenstein 
in der Entwicklung des Studiums von Frauen an der Universität Zürich. In Europa hatte nur Paris den Frauen die Pforten seiner Universität noch einige Jahre früher geöffnet.

1868 immatrikulierte sich Marie Vögtlin als erste Schweizerin und 10. Medizinstudentin an der Medizinischen Fakultät der Universität Zürich. Sie legte 1872 das medizinische Staatsexamen ab. Da in Zürcher Spitälern damals keine weiblichen Assistierenden angestellt wurden, musste sie für ihre Weiterbildung nach Leipzig und Dresden gehen, wo sie in der Geburtshilfe promovierte. 1874 kehrte sie nach Zürich zurück und heiratete den Geologieprofessor Albert Heim. Von 1874 bis zu ihrem Tod im Jahr 1916 führte sie eine frauenärztliche Praxis an der Hottingerstrasse in Zürich. Marie Heim-Vögtlin gelang als erster Schweizer Ärztin eine sogenannte «double career»: Parallel zu ihrer beruflichen Karriere als Gynäkologin erlangte sie auch als Mutter von zwei Kindern und Familienfrau hohes Ansehen.

Anna Heer, etwa 20 Jahre jünger als Marie Vögtlin, musste nicht mehr um die Zulassung zum Medizinstudium kämpfen. Ihr besonderes Interesse galt der Krankenpflege. Sie setzte sich für ein Lehrkrankenhaus für Pflegerinnen ein. Dieses Spital wurde über viele Jahre nur von Frauen geführt. Anna Heer war nach ihrer Weiterbildung die erste chirurgisch tätige Ärztin der Schweiz und überhaupt die erste Chefärztin: sie leitete von 1901 bis 1918 das Spital Pflegerinnenschule in Zürich-Hottingen.

Wenn man die Lebensläufe der ersten Medizinerinnen betrachtet, fallen trotz aller Verschiedenheiten einige Gemeinsamkeiten auf: Der Mut, sich gegen Konventionen und Barrieren aufzulehnen, und ihr Durchhaltewille in der Verwirklichung ihrer Ziele. Viele setzten sich später für soziale Probleme ein und kämpften mit Tinte und Taten für diese Anliegen.

Die Universität Zürich hat in der Geschichte des Frauenstudiums gesamteuropäisch eine bemerkenswerte Vorreiterrolle gespielt. Die Ärztinnen, welche hier ausgebildet wurden, praktizierten später in vielen Ländern.

Im Ausland herrschte damals mehrheitlich noch eine ganz andere Gesinnung. So rechtfertigte der Münchener Anatomie- und Physiologieprofessor T. L. von Bischoff den Ausschluss der Frauen vom Medizinstudium mit dem geringeren Hirngewicht der Frauen; er schloss daraus auf eine mindere Befähigung zur geistigen Beschäftigung. In einer 1872 erschienenen Schrift führte von Bischoff aus, dass er sich durch den Anblick einer am Kadaver präparierenden Frau unangenehm berührt fühlte. Dass es aber nicht nur um Fragen der Schicklichkeit, sondern auch um die Ausübung von Macht ging, macht folgendes Zitat deutlich:

«Man denke sich eine Frau als ärztliche Dirigentin eines Hospitals, oder als Gerichtsärztin! Muss nicht jeder bei dem Gedanken lachen (oder auch weinen), dass eine Frau, selbst wenn sie die medizinischen Kenntnisse dazu hätte, den hohen Grad von Autorität ausüben soll, welcher dem Dirigenten eines Spitals unentbehrlich ist?» [1]
Kriterium war also nicht die medizinische Befähigung, sondern die Machtposition, die von Bischoff den Frauen auf keinen Fall zugestehen wollte. Gerade weil die Frauen auf dem Terrain von Gesundheit und Krankheit Kompetenz und Autorität besassen, mussten sie als Konkurrentinnen auf Distanz gehalten werden.

Trotz der liberalen Haltung der offiziellen Zürcher Universitätsorgane standen nicht wenige Professoren dem Experiment Frauenstudium am Ende des 19. Jahrhunderts ambivalent und kritisch gegenüber. Der Zürcher Physiologieprofessor Ludimar Hermann formulierte 1872 seine Bedenken in einem Aufsatz folgendermassen:

"Es könnte das grenzenlose Unglück entstehen, welches die Universität vernichten würde, dass nämlich die Zahl der Studentinnen grösser würde als die der Studenten. Die Universität Zürich wird sicher, trotz ihrer jetzigen Blüte, daran zugrunde gehen, wenn nicht entschiedene Schritte geschehen.» [2]

Die Entwicklung der Studierendenzahlen an der Universität Zürich widerlegen Hermanns Hypothese (Tabelle 1). In der Medizin sind schon seit 1995 mehr als die Hälfte der Studienanfänger Frauen. Im Herbst 2000 lag der Frauenanteil bei 63 \% [3]. Ähnliche Entwicklungen zeigen sich an den anderen Schweizer Universitäten [4], aber auch in den meisten anderen westeuropäischen Ländern [5] und in den USA [6].

\section{Tabelle 1}

Studienbeginnende im Fach Humanmedizin an der Universität Zürich 1990-2000.

\begin{tabular}{lrrrrrr}
\hline & 1990 & & 1995 & \multicolumn{3}{r}{2000} \\
\hline Frauen & 110 & $48 \%$ & 126 & $51 \%$ & 106 & $63 \%$ \\
Männer & 120 & $52 \%$ & 123 & $49 \%$ & 63 & $37 \%$ \\
Total & 230 & & 249 & & 169 & \\
\hline
\end{tabular}

Betrachtet man das Geschlechterverhältnis der Studierenden aller Fakultäten der Universität Zürich (Tabelle 2), waren im WS 2000/01 erstmals mehr Frauen als Männer (51 versus 49\%) immatrikuliert. Die Zukunft wird zeigen, ob die Universität Zürich entsprechend der Prophezeiung von Ludimar Hermann ihrem Ende entgegengeht oder ob sie dank ihres hohen Anteils an Studentinnen und hoffentlich bald auch Dozentinnen vor einer neuen Blütezeit steht.

\section{Tabelle 2}

Geschlechterverhältnis aller Studierenden der Universität Zürich 1990-2000.

\begin{tabular}{lrrrrrr}
\hline & 1990 & & 1995 & & 2000 & \\
Frauen & 9310 & $44 \%$ & 7616 & $47 \%$ & 10530 & $51 \%$ \\
Männer & 11868 & $56 \%$ & 8613 & $53 \%$ & 10087 & $49 \%$ \\
Total & 21178 & & 16229 & & 20617 & \\
\hline
\end{tabular}




\section{Entwicklung der Berufstätigkeit von Ärztinnen und Ärzten in der Schweiz}

Nach dem Abbau der Zugangsbarrieren für Frauen sowohl an den Gymnasien wie auch an den Universitäten nahmen zunehmend mehr Frauen ein Studium auf. Welche Entwicklung nahm in den folgenden Jahrzehnten die Zahl der Studienabschlüsse von Frauen in der Medizin?

Die gesamtschweizerische Statistik zur prozentualen Verteilung der Diplomierungen von Ärztinnen und Ärzten von 1880 bis 1999 zeigt (Abb. 1), dass sich der Anteil an diplomierten Ärztinnen bis 1960 bei etwa 10\% einpendelte. Seit $1960 \mathrm{kam}$ es zu einer steten Zunahme von diplomierten Ärztinnen bis auf 45,4\% im Jahr 1999. Gleichzeitig nahm die Zahl der diplomierten Ärzte seit 1975 kontinuierlich ab [7, 8].

\section{Abbildung 1}

Anzahl Diplomierungen von Ärztinnen und Ärzten in der Schweiz von 1880 bis 1999.

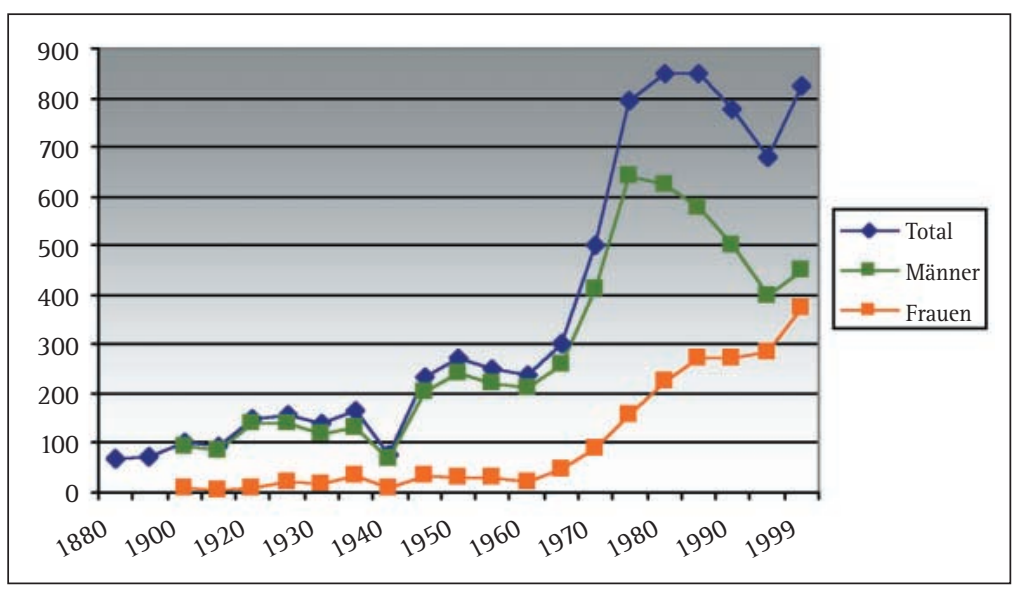

Dieser Trend wird sich in der Schweiz wie auch in den anderen westlichen Ländern in den kommenden Jahren fortsetzen. In den osteuropäischen Ländern wird die Medizin schon seit Mitte des 20. Jahrhunderts mehrheitlich von Frauen ausgeübt.

Wie gestaltet sich die weitere berufliche und persönliche Karriere von jungen Ärztinnen und Ärzten nach der Diplomierung?

Im gesellschaftlichen Bewusstsein haben sich die Einstellung zur Mutterrolle und der Anspruch nach ausschliesslicher Betreuung der Kinder durch die Mutter verändert. Dies trägt sicherlich mit dazu bei, dass sich auch auf institutioneller Ebene Veränderungen im Sinne einer Flexibilisierung der Anstellungsbedingungen vollziehen können. In der Schweiz kann seit 1992 die ärztliche Weiterbildung in Teilzeit bei einer Mindestanstellung von 50\% absolviert werden [9]. Allerdings liegt es in der Entscheidungsbefugnis der jeweiligen Fachgesellschaften, ob die ganze Weiterbildungszeit (wie z. B. für die Facharztqualifikation in Psychiatrie oder Sozial- und Präventivmedizin) oder nur ein gewisser Anteil davon in Teilzeit abgeleistet werden kann. Die genannte Flexibilisierung wirkt sich auch auf die Berufstätigkeit von Ärztinnen und Ärzten und deren Weiterbildungsqualifikationen aus.

Im Jahr 1999 waren in der Schweiz total 24026 Ärztinnen und Ärzte berufstätig, davon waren 28,4\% Frauen und dementsprechend 71,6\% Männer [8]. Dass der Anteil berufstätiger Ärztinnen nicht höher ist, liegt daran, dass bei den älteren Ärzten die Männer überrepräsentiert sind. In Deutschland beträgt der Frauenanteil 37\% [10], in den USA 26\% [11].

Auch bei den Facharztqualifikationen nahm der Anteil bei den Schweizer Ärztinnen auf über 51\% zu [8]. In Deutschland haben bereits 56\% der Ärztinnen eine Gebietsbezeichnung erworben [10]. Von den Schweizer Ärzten haben 83\% einen Facharzttitel [8].

Diese Zahlen machen deutlich: die Feminisierung des Arztberufes schreitet fort. Es stellt sich jedoch die Frage, ob diese Entwicklung auch schon zu einem höheren Frauenanteil in leitenden ärztlichen Positionen geführt hat? Die im folgenden beschriebenen Daten von Zürich sind entsprechend verschiedener Studien mit Zahlen und Verhältnissen im übrigen europäischen und amerikanischen Raum vergleichbar.

Eine Untersuchung zur "Gleichberechtigten Nachwuchsförderung von Ärztinnen und Ärzten am UniversitätsSpital Zürich" von Jacquemart und Boos [12] zeigt, dass mit steigender Hierarchiestufe der Frauenanteil deutlich abnimmt. Finden wir bei den Assistierenden noch 40\% Frauen, so schrumpft ihr Anteil auf Stufe Oberärztinnen/Oberärzte auf 30\%, bei den Leitenden Ärztinnen und Ärzten sind es nur 8\% und unter den Klinikdirektoren finden sich keine Frauen mehr. Weiterhin zeigten sich folgende Ergebnisse:

- Karriereförderung ist nicht institutionalisiert und folglich wenig transparent.

- Frauen schätzen die Karriereförderung durch ihre Vorgesetzten tiefer ein und erhalten weniger Karriereangebote als ihre männlichen Kollegen.

- Weibliche Assistierende oder Oberärztinnen erleben die organisatorischen Klinikstrukturen als autoritärer.

- Ärztinnen wenden mehr Zeit für die Patientenbetreuung auf als ihre männlichen Kollegen. Es gilt das Motto: «Frauen spielen Männer für die Forschung frei!» Die klinische Arbeit hat jedoch vergleichsweise wenig Bedeutung auf einem Karriereweg nach oben.

Eine amerikanische Studie weist nach, dass Ärztinnen viermal weniger ärztliche Kunstfehler machen als ihre männlichen Kollegen. Als Grund fanden sie, dass Frauen mehr Zeit mit den Patienten verbringen. Dies erhöht ihr Verständnis, verhindert Fehler, ist aber ein Karrierehemmer [13].

Die ungleichen Karrierechancen werden auch aus anderen Ländern berichtet. Selbst eine Regierungsunterstützung zur Gleichstellung von Mann und Frau, wie sie in Norwegen lanciert wurde, konnte den Anteil an Chefärztinnen nur unwesentlich erhöhen [14]. Eine amerikanische Studie [15] sieht als Hauptgrund für die Unterrepräsentanz von Frauen in Führungspositionen den Einfluss von unbewussten, 
nicht ausgesprochenen Geschlechterstereotypen. Die gängigen Stereotype halten Frauen für fürsorglich, gefühlsbetont und führungsschwach, während Männer als autonom, selbstbewusst, sach- und lösungsorientiert gelten. Aus der Sozialpsychologie ist bekannt, dass Geschlechterstereotype die Wahrnehmung von sich selbst und vom anderen Geschlecht systematisch beeinflussen, und zwar in der Weise, dass sich Männer in der Regel in ihrer Kompetenz über- und Frauen unterschätzen. Diese fixierten Einstellungen generieren vor allem für Frauen Nachteile, die sich im Verlauf aufsummieren und schlussendlich zum Phänomen der "gläsernen Decke» [16] beitragen. Darunter versteht man unsichtbare Barrieren, wenn frau auf der Karriereleiter nach oben strebt.

Welche Chancen haben Ärztinnen und Ärzte im Hinblick auf eine akademische Laufbahn? Trotz der grossen Zahl gut ausgebildeter Ärztinnen ist ihnen der Zugang zu einer akademischen Laufbahn und entsprechenden akademischen Position nicht in gleichem Mass gelungen wie ihren männlichen Kollegen. Aus den Jahresberichten der Universität Zürich der letzten 10 Jahre geht hervor, dass von den 231 Habilitierenden im Fach Humanmedizin nur 20 Frauen, das sind 8,7\%, der Titel einer Privatdozentin verliehen wurde.

Auch bei den akademischen Positionen zeigt sich im Jahr 2000 eine ähnliche Untervertretung. Nur eine Professorin hat ein Ordinariat inne. Bei den Extraordinariaten liegt der Frauenanteil bei 13\%, bei den Titularprofessuren bei 5\%, von den Habilitierten sind $9 \%$ Frauen. Selbst auf der karrieremässig niedrigeren Stufe der Lehrbeauftragten finden sich nur 21\% Frauen. Ob die Untervertretung der Frauen mit einer mangelnden Förderung, mit anderen Lebensentwürfen oder mit den Organisationsstrukturen der einzelnen Institutionen zusammenhängt, lässt sich nicht mit Bestimmtheit sagen.

Wie erwähnt finden sich an den anderen Universitäten der Schweiz und im Ausland vergleichbare Verhältnisse.

Ein wesentlicher die Karriere beeinflussender Faktor ist die persönlich gewählte Lebensform, die sich in Abhängigkeit vom Geschlecht hinderlich oder fördernd auswirken kann. Entsprechend einer aktuellen soziologischen Studie aus der Schweiz [17] sind von den Angehörigen der Professorenschaft und des oberen Mittelbaus an Schweizer Medizinischen Fakultäten 90\% der Männer verheiratet, hingegen nur die Hälfte der Frauen. Alleine leben 10\% der Ärzte, aber immerhin ein Viertel der Frauen. Ärzte haben im Vergleich zur Durchschnittspopulation eher mehr Kinder, während verheiratete oder in Partnerschaft lebende Ärztinnen entweder keine oder maximal ein Kind haben. Ähnliche Zahlen werden von Autoren aus den USA, aus Kanada und Grossbritannien berichtet [18-20]. Das heisst, Partnerschaft oder Familie bilden für Ärztinnen ein Karrierehindernis, während Ärzte durch ihre Familien in der Verwirklichung ihrer Karriere eher unterstützt werden. Dies hängt auch damit zusammen, dass Ärzte häufiger mit Partnerinnen zu- sammenleben, die im Hinblick auf Ausbildung und Status etwas unter ihnen stehen und deshalb eher bereit sind, ihre beruflichen Ambitionen hinter die des Mannes zurückzustellen. Ärztinnen wählen beruflich eher gleichrangige Partner. Hier stellt sich häufig die Frage, wie eine "double career» verwirklicht werden kann, wenn auch noch Kinder zu versorgen sind. In der Regel wählen Ärztinnen mit Kindern eine Unterstützung durch bezahlte Haushaltskräfte, während Ärzte ihre Ehefrau als wichtigsten "caretaker» angeben [19, 21].

\section{Determinanten für die Karriereentwicklung junger Ärztinnen und Ärzte}

Die bisherigen Ausführungen weisen darauf hin, dass trotz gleicher Zugangschancen für Frauen und Männer in der weiteren beruflichen Entwicklung ein geschlechtsabhängiger Segregationsprozess einsetzt. Dieser Prozess lässt sich aus der "Theorie der Strukturierung" von Giddens [22] ableiten: Sie geht davon aus, dass zwischen Individuum, d.h. Arzt bzw. Ärztin, und Organisation, wie sie z.B. ein Spital darstellt, eine Interaktion besteht.

Bis heute haben mehrheitlich Männer die Führungspositionen in Spitälern und Instituten inne. Entsprechend ihrer Sozialisation bevorzugen sie eher einen "comand and control style of managing others" [23] und erachten eine 150\%ige Arbeitsbelastung als selbstverständlich. Dies hat zur Folge, dass sich wieder mehrheitlich Männer in solchen hierarchischen Strukturen behaupten und einen Weg nach oben antreten. Auf diese Weise perpetuieren sich patriarchale Organisationsstrukturen. Frauen bedienen sich in leitenden Positionen eher einer "interactive leadership» [23]. Ausserdem bevorzugen sie integrierte Lebensentwürfe, d.h. dass den ausserberuflichen Belangen ebenfalls Wichtigkeit beigemessen wird [24]. Dies hat zur Folge, dass Frauen häufiger medizinische Institutionen vorzeitig verlassen, in denen Konkurrenz statt Kooperation vorherrschen und die Arbeit am Patienten weniger gilt als eine Forschungstätigkeit, die häufig noch in der Freizeit geleistet werden muss. Das heisst, Ärztinnen führen lieber eine eigene Praxis, in der sie ihren eigenen Führungsstil praktizieren können und genügend Spielraum für eine Vereinbarkeit von Beruf und ausserberuflichen Aufgaben haben. Wenn also Frauen schon auf einer karrieremässig tieferen Stufe das Spital verlassen, verliert eine Institution nicht nur wertvolle «human resources", sondern auch ein Innovationspotential [12]. Insofern bedingen sich "doing gender» und "doing organization" wechselseitig [25]. Die Gefahr besteht, dass nicht die "Besten" an den grossen Spitälern bleiben oder eine akademische Karriere durchlaufen, sondern jene, die sich am besten mit den gegebenen Strukturen arrangieren.

Die genannten Ausführungen verdeutlichen, welchen Einfluss Chefärztinnen und Chefärzte auf die ärztliche Weiterbildung und vor allem auf die Karriereentwicklungen von jungen Ärztinnen und 
Ärzten ausüben. Deshalb haben wir im Rahmen eines vom Schweizerischen Nationalfonds geförderten Forschungsprojekts im Herbst 2000 alle Chefärztinnen und Chefärzte der drei Deutschschweizer Universitätskantone Basel, Bern und Zürich angeschrieben und um die Beantwortung von einigen Fragen gebeten.

Die wichtigsten Ergebnisse dieser Umfrage, an welcher sich 189 Chefärzte und 18 Chefärztinnen beteiligten (Rücklaufquote knapp 40\%) lassen sich in folgenden Feststellungen zusammenfassen:

- Für eine Karriere fördernd gelten an Persönlichkeitsmerkmalen: Berufliches Engagement, wissenschaftliches Interesse, soziale Kompetenz sowie Zielstrebigkeit, Durchhaltevermögen und Charakterstärke.

- Von seiten der Institution scheinen den Chefärzten und Chefärztinnen folgende Rahmenbedingungen karrierefördernd: Gute Qualität der Ausbildung, gutes Arbeitsklima, gezielte Karriereförderung, Renommee der Klinik und eine günstige Klinikstruktur.

Die Einschätzung von Chefärzten und Chefärztinnen unterscheiden sich nur tendenziell, indem erstere die fachlichen Aspekte und letztere die persönliche Motivation und das Arbeitsklima etwas höher bewerteten.

Da bisher nur Querschnittuntersuchungen $\mathrm{zu}$ karrierefördernden und -hinderlichen Faktoren vorliegen, führen wir derzeit eine prospektiv angelegte Studie bei den Staatsexamenskandidatinnen und -kandidaten im Fach Humanmedizin der drei Deutschschweizer Universitäten durch. Es soll untersucht werden, in welcher Weise persönliche, institutionelle und gesellschaftliche Faktoren die weitere berufliche Entwicklung beeinflussen.

Die relativ hohe Rücklaufquote von $72 \%$ bei der Erstbefragung im Herbst 2000 und zahlreiche Kommentare zeigen das grosse Interesse der Studierenden an der Thematik. Unter anderem wurden die Teilnehmenden nach verschiedenen Aspekten ihrer beruflichen Karrieremotivation gefragt. Dabei werden drei Aspekte unterschieden: eine sog. intrinsische Karrieremotivation, d.h. das fachorientierte Engagement und der innere Antrieb; eine sogenannte extrinsische Karrieremotivation, die sich eher auf taktische Überlegungen zum Erreichen eines Karriereziels und auf Macht und Prestige im Beruf bezieht. Der dritte Bereich umfasst die extraprofessionellen Belange. Mit diesen Fragen wird der Stellenwert von ausserberuflichen Interessen, vor allem Freizeit und Familie, im Vergleich mit den beruflichen Interessen erhoben. Für eine zukünftige berufliche Karriere im Sinne von Führungsverantwortung und innovativem Potential hat die intrinsische Karrieremotivation die grösste Bedeutung.

Erste Auswertungen zeigen, dass Studierende mit hoher intrinsischer Karrieremotivation ihre Dissertation bereits abgeschlossen und wissenschaftlich publiziert haben, sie haben an medizinischen Kongressen teilgenommen, ihre zukünftigen Assistierenden- stellen abgemacht und interessieren sich eher für eine klinische und/oder akademische Laufbahn. Sie haben einen Mentor oder eine Mentorin, welche sie während des Studiums und im Hinblick auf ihre berufliche Laufbahn beraten. Es zeigen sich keine Geschlechtsunterschiede. Das heisst, angehende Ärztinnen und Ärzte streben gleich häufig eine Karriere in der Medizin an. Möglicherweise definieren jedoch Frauen und Männer den Begriff Karriere in unterschiedlicher Weise. Es finden sich auch keine Zusammenhänge zwischen Karrieremotivation und Ausbildungsniveau der Eltern oder der Herkunft aus einer Arztfamilie. Die Vorbildfunktion aus der Herkunftsfamilie spielt vermutlich eine geringe Rolle. Entgegen unserer Hypothese haben Studierende mit hoher Karrieremotivation sich am Ende des Studiums noch nicht häufiger entschieden, welche Facharztweiterbildung sie anstreben, als diejenigen mit niedriger Karrieremotivation.

Es ist geplant, die Studienteilnehmenden alle 2 Jahre während ihrer Weiterbildungszeit zu ihrer beruflichen und persönlichen Entwicklung zu befragen. Im Längsverlauf werden sich dann wahrscheinlich Prädiktoren für unterschiedliche berufliche Biographien und persönliche Lebensentwürfe zeigen.

\section{Ausblick}

Kehren wir zu den eingangs gestellten Fragen zurück. Welche Faktoren tragen zu einer "Feminisierung der Medizin" bei? Und wie sollten sich die Bedingungen für eine Karriere in der Medizin verändern, damit sie für Frauen und Männer in gleicher Weise attraktiv und erstrebenswert ist?

\section{Soziokultureller Wandel des Arztberufes}

Auf der soziokulturellen Ebene spielen vor allem zwei gegenläufige Entwicklungen eine wichtige Rolle: Einerseits ein Trend zur weitreichenden Professionalisierung des Arztberufes; andererseits ein Trend $\mathrm{zu}$ einer fortschreitenden "Deprofessionalisierung" durch die "Dominanz der Konsumenten" und einem «Hierarchieverlust der Anbieter» [26].

\section{Auswirkungen der Professionalisierung}

Die zeitaufwendige und zunehmende Reglementierung der ärztlichen Weiterbildung, die hohen Anforderungen für die jeweils nächsten Karriereschritte und die Unsicherheit, ob sich der hohe Einsatz schliesslich lohnt, schreckt schon bei der Studienwahl immer mehr junge Männer von der Medizin ab. Sie entscheiden sich eher für Berufe im Bereich der Wirtschaft oder der neuen Technologien, in denen sie schneller ihre Karriere vorantreiben können und grössere Gestaltungs- und Einkommensmöglichkeiten haben. Offensichtlich sind Frauen weniger auf schnellen Erfolg, Prestige und hohes Einkommen fixiert. Sie sind wahrscheinlich auch weniger auf das Erreichen einer bestimmten Karriereposition ausgerichtet, als dass sie vor allem Freude und Erfüllung in ihrer beruflichen Tätigkeit suchen. 


\section{Auswirkungen der "Deprofessionalisierung"} Der leichte Zugang für Laien zu neuem medizinischem Wissen über die elektronischen Medien verringert die Wissenskluft zwischen Ärztinnen/Ärzten und Patientinnen/Patienten. Dies führt dazu, dass gut informierte "Kunden» ihre Ärzte mit einer ähnlichen Haltung konsultieren, wie sie dies bei der Nachfrage nach anderen Dienstleistungen tun, d.h. Arztpraxis und Spital werden als "Gesundheitsmarkt" betrachtet: Ärztinnen und Ärzte bieten ihr fachliches Wissen, ihre therapeutischen Angebote und ihre durch Medizintechnologie optimierte Handlungskompetenz an, und der "Konsument" vergleicht die verschiedenen Angebote bezüglich Qualität und Preis. Frauen haben bisher nur zu einem kleinem Teil höhere Kaderpositionen eingenommen und sind eher dahingehend sozialisiert, Dienstleistungen zu erbringen.

\section{Institutionelle Faktoren für gleichberechtigte Karrierechancen}

Neben den soziokulturellen Faktoren prägen auch institutionelle Faktoren die berufliche Entwicklung. Damit Ärztinnen und Ärzte gleichberechtigte Bedingungen für eine Karriere in der Medizin haben, bedarf es einiger struktureller Veränderungen.

Hier geht das UniversitätsSpital Zürich neue Wege. Basierend auf den vorher zitierten Ergebnissen der Untersuchung zur «Gleichberechtigten Nachwuchsförderung von Ärztinnen und Ärzten am UniversitätsSpital Zürich» [12] arbeitet derzeit eine Arbeitsgruppe verschiedene Projekte aus in den Bereichen Mentoring, neue Arbeitszeitmodelle, Angebot von Kleinkinderbetreuung und Gleichstellungscontrolling.

Abschliessend stellt sich die Frage: Welche Frauen und Männer braucht die Medizin in der Zukunft?

\section{Zukunftsperspektiven von Frauen und Männern in der Medizin}

Wie man aus sozialpsychologischen Untersuchungen weiss, unterscheiden sich Männer und Frauen in ihrem Kommunikations- und Interaktionsstil. In männerdominierten Gruppen beobachtet man einen konkurrierenden, in frauendominierten Gruppen einen kooperativen Kommunikations- und Interaktionsstil. In Zukunft werden Frauen und Männer gleichrangig auf allen Ausbildungs- und Hierarchiestufen des Medizinalbetriebs tätig sein. Es geht dann nicht darum, dass Frauen als «Supermänner» männlichen Karriere- und Lebenskonzepten nacheifern oder dass nur noch solche Männer Ärzte werden, welche die Freude an Kompetition und Prestige verloren haben. Dies würde nur eine Umkehrung der Geschlechterstereotype bedeuten.

Anzustreben sind vielmehr strukturelle Rahmenbedingungen und ein Arbeitsklima, die partizipative Kooperation und kreative Konkurrenz ermöglichen und damit Voraussetzungen für ein hohes wissenschaftliches Niveau und eine patientengerechte Krankenversorgung liefern.

Die Rollen von Frau und Mann in unserer Gesellschaft wandeln sich. Die traditionelle Aufteilung in «breadwinner» und "caretaker» ist überholt. Man kann sich heute durchaus eine Frau als «Dirigentin eines Spitals» vorstellen, aber auch einen Mann, der um 18 Uhr die Arbeit in der Klinik beendet, weil er sein Kind von der Krippe abholt und den Abend mit der Familie verbringt. Immer mehr jüngere Ärztinnen und Ärzte planen einen integrierten Lebensentwurf, d.h. eine Gleichrangigkeit von beruflicher Karriere und persönlichem Lebensstil. Diese Grundeinstellung bringt auch Vorteile für die Kunden, indem "Curing" und "Caring" [27] zu gleichrangigen Zielen in der Behandlung und Betreuung von Patienten werden.

\section{Literatur}

1 von Bischoff TL. Das Studium und die Ausübung der Medicin durch Frauen. München; 1872.

2 Hermann L. Das Frauenstudium und die Interessen der Hochschule Zürich. Zürich; 1872.

3 Statistik der Universität Zürich 1990-2000.

Zürich: Abteilung Informationsmanagement \& Controlling Universität Zürich; 2001.

4 Bundesamt für Statistik. Statistik zu Studierenden der Humanmedizin in der Schweiz. Sektion Hochschulen und Wissenschaft. Bern; 2000.

5 Statistisches Bundesamt Deutschland. Statistik zu Studierenden der Humanmedizin in Deutschland. Wiesbaden; 2001.

6 American Medical Association. Statistics of Medical Students in the United States. Chicago, Ill.; 1998.

7 Verbindung der Schweizer Ärztinnen und Ärzte FMH. Statistik der Diplomierungen von Ärztinnen und Ärzten von 1880-1999. Bern: FMH; 2000.

8 FMH-Ärztestatistik 1999. Schweiz Ärztezeitung 2000; 81(16):816-33.

9 Weiterbildungsordnung, FMH, 1992, Revision 1998. Bern: FMH.

10 Bundesärztekammer Deutschland. Berufstätige Ärztinnen und Ärzte zum 31.12.1999. Wiesbaden; 2001.

11 American Medical Association. Physician Characteristics and Distribution in the U.S. until 1998. Edition and prior editions. Chicago, Ill.; 2000/2001.

12 Jacquemart C, Boos L. Gleichberechtigte Nachwuchsförderung von Ärztinnen und Ärzten am UniversitätsSpital Zürich. Institut für Betriebswirtschaftliche Forschung der Universität Zürich, Diskussionsbeitrag Nr. 50. 2000.

13 Taragin MI, Wilzek AP, Karns ME, Trout R, Carson JL. Physician demographics and the risk of medical malpractice. Am J Med 1992;93:537-42.

14 Kvaerner KJ, Aasland OG, Botten GS. Female medical leadership: cross sectional study. Br Med J 1999;318:91-4.

15 Valian V. Why so slow? The advancement of women. Cambridge, Massachusetts: MIT Press; 1999.

16 Franck E, Jungwirth C. Vorurteile als Karrierebremse? Ein Versuch zur Erklärung des Glass Ceiling-Phänomens. Zeitschrift für betriebswirtschaftliche Forschung 1998;50(12):1083-97.

17 Leemann RJ. Geschlechtsspezifische Unterschiede in den Bildungs- und Berufsverläufen von Wissenschaftlerinnen und Wissenschaftlern in der Medizin. Empirische Ergebnisse einer Nationalfondsstudie an Schweizer Hochschulen. Vortrag. Symposium: Geschlechterforschung in der Medizin. Jahreskongress 2000 der Schweizerischen Gesellschaft für Psychosoziale Medizin, Zürich. 2000.

18 Limacher MC, Zaher CA, Walsh MN, Wolf WJ, Douglas PS, Schwartz JB et al. The ACC professional life survey: career decisions of women and men in cardiology. J Am Coll Cardiol 1998;32:827-35.

19 Heathcote J, Cauch-Dudek K, Rhyne D. The professional lives of women in gastroenterology: a Canadian comparison study with men. Gastroenterology 1997;113:684-6. 
20 Davidson JM, Lambert TW, Goldacre MJ. Career pathways and destinations. 18 years on among doctors who qualified in the United Kingdom in 1977: postal questionnaire survey. Br Med J 1998;317:1425-8.

21 Dresler CM, Padgett DL, Mackinnon SE, Patterson GA. Experiences of women in cardiothoracic surgery. Arch Surg 1996;131:1128-34.

22 Giddens A. Die Konstitution der Gesellschaft. Grundzüge einer Theorie der Strukturierung. Frankfurt, New York: Campus; 1992.

23 Rosener JB. Ways women lead: In: Kanter RM. Reach for the top. Boston: Harvard Press; 1990. p. 13-23.

24 Wetterer A. Theoretische Entwicklungen der Frauen- und Geschlechterforschung über Studium, Hochschule und Beruf ein einleitender Rückblick. In: Neusel A, Wetterer A.

Vielfältige Verschiedenheiten: Geschlechterverhältnisse in Studium, Hochschule und Beruf. Frankfurt, New York: Campus; 1999. S. 15-35.
25 Osterloh M, Wübker S. Wettbewerbsfähiger durch Prozessund Wissensmanagement. Mit Chancengleichheit auf Erfolgskurs. Wiesbaden: Gabler; 1999. S. 201-14.

26 Freidson E. The reorganization of the medical profession. Med Care Rev 1985;42:11-35.

27 von Castelberg B. Caring statt Curing. Vortrag: Feminisierung in der Medizin - Chance oder Risiko? Am Beispiel der Frauenheilkunde. Symposium BerlinNeukölln. 2001. 